\title{
Correction
}

\section{Correction: Horikawa and Ojima. Cortical Activation Patterns Evoked by Temporally Asymmetric Sounds and Their Modulation by Learning (eNeuro March/April 2017, 4(2) e0241-16.2017 1-19 https://doi.org/10.1523/ENEURO.0241-16.2017)}

In the article "Cortical Activation Patterns Evoked by Temporally Asymmetric Sounds and Their Modulation by Learning" by Junsei Horikawa and Hisayuki Ojima, which appeared on e0241-16.2017 of the March/April 2017 issue, authors provided a misleading legend for Figure 6 . Since the figure includes not only response traces evoked by $F$ but also those evoked by revF, the description "evoked by the first F segment" in the second sentence should be changed to "evoked by the first segment." This correction would help readers understand differences in response signals evoked by the temporally asymmetric sounds. Figure 6 legend has been corrected on the online PDF version and displayed below.

Figure 6. Trial-unique response traces recorded at the maxP. Temporal traces of the response signals (dF/ $\left.F_{\text {max }}, \%\right)$ evoked by the first segment at the channels where the spatiotemporal maxP within the $\mathrm{Al}$ is evoked. The sound waveforms below traces show the delay times and duration of the stimulus sounds ( $F$ and revF). The time of maxP is indicated by the dotted line for each trace. $F$, normal natural sound. revF, time-reversed version of $F$. 